\title{
Automated Spatial Alignment of Video Frame Data from In Situ Experiments.
}

\author{
Joshua D. Sugar ${ }^{1}$, Benjamin W. Jacobs ${ }^{2}$, Aron W. Cummings ${ }^{1}$, and David B. Robinson ${ }^{1}$ \\ 1. Sandia National Laboratories, Livermore, CA, USA. \\ 2. Protochips, Inc. Raleigh, NC, USA.
}

A fairly simple operation to perform on frames of video data is to translate them so that features are aligned in adjacent frames. Because of the vast quantity of data generated while recording video during an in situ experiment, it is necessary that this alignment procedure be automatic and requires little operator intervention. In addition, because of the variety of manufacturer, software, and accessory combinations available for electron microscopes, it is beneficial to have an algorithm that can postprocess data and be independent of acquisition platform. Here, we will show a readily available Matlab script that can perform this alignment function.

A cross-correlation method for calculating the shift needed to align two images is relatively straightforward [1]. Basically, the Fast-Fourier Transform (FFT) of two images is multiplied together. The position of the maximum of the product of these two images is the amount that one image must be shifted relative to the other for alignment. A number of commercially available software products do this, for example, when acquiring a spectrum image (e.g. FEI's TIA, Gatan's Digital Micrograph, Oxford's INCA, Bruker's Espirit, just to name a few). However, our script is platform independent, free, and can accept as input a variety of customary video and image formats. An instruction manual for this script will be available soon [2], and we will provide information on how to obtain the script [3]. Here, we will present a brief description of how to use the script and the resulting output.

Figure 1 shows the measured drift from two types of in situ heating holders. While the magnitude of the drift is lower in a microfabricated heater [4], the magnitude of the drift is sufficient that at high magnification a region of interest could move out of the field of view. Figure 2 shows the result of translating image frames to align features using our script.

\section{References:}

[1] J.C. Russ, The image processing handbook, CRC Press, Boca Raton, 1994.

[2] J.D. Sugar, et al., Microscopy Today (submitted)

[3] http://www.mathworks.com/matlabcentral/fileexchange/45453-driftyshiftydeluxe-m

[4] J. Damiano, et al., Microscopy and Microanalysis 14 (2008)

[5] Sandia National Laboratories is a multi-program laboratory managed and operated by Sandia Corporation, a wholly owned subsidiary of Lockheed Martin Corporation, for the U.S. Department of Energy's National Nuclear Security Administration under contract DE-AC04-94AL85000. 


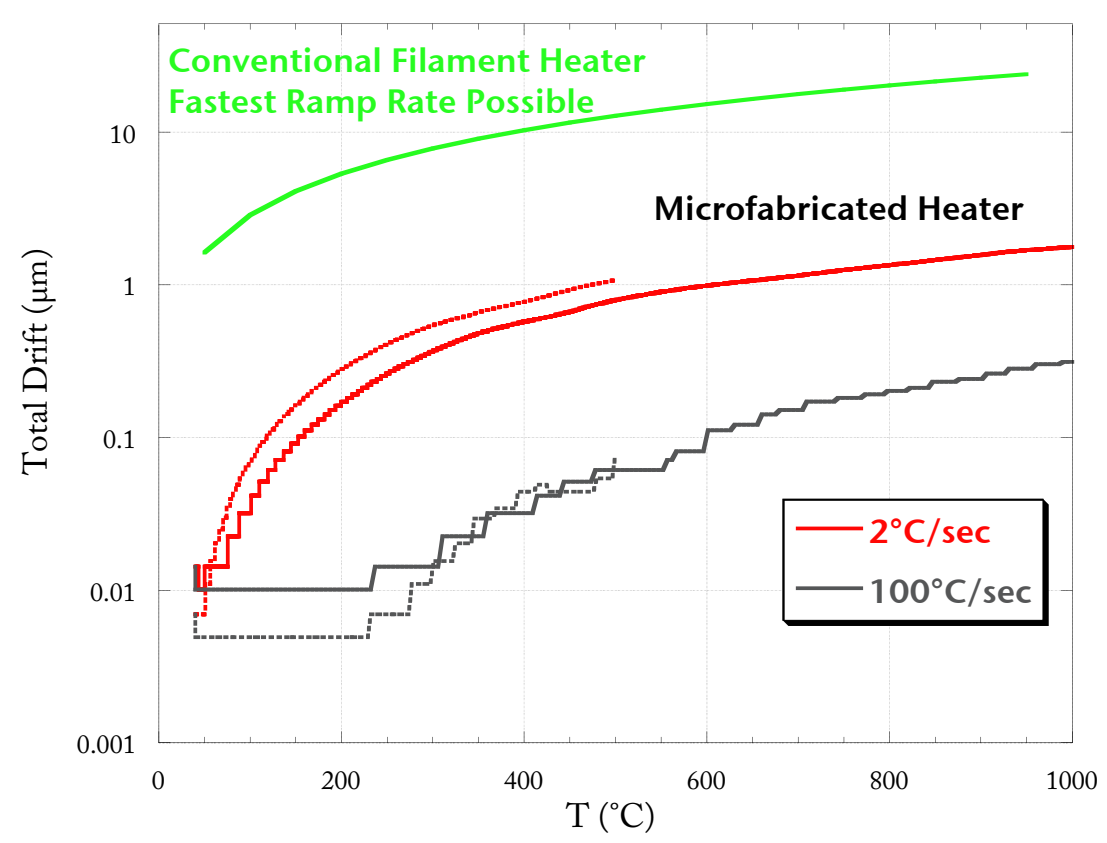

Figure 1. Spatial drift measured during in situ heating experiments using our script. The magnitude of the drift is large enough that a drift-correction routine is required to keep the feature of interest in the center of the field of view.

\section{Unprocessed Video Frames}

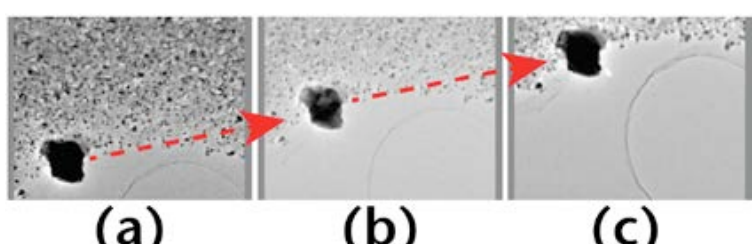

\section{Drift-Corrected Video Frames}

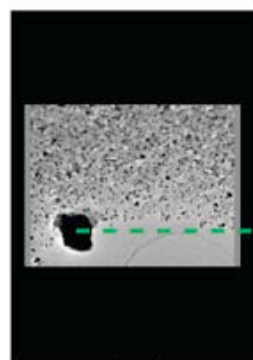

(d)

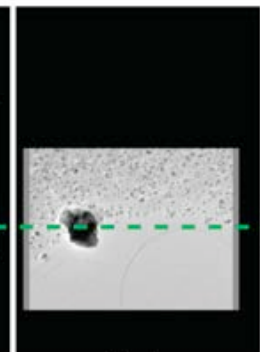

(e)

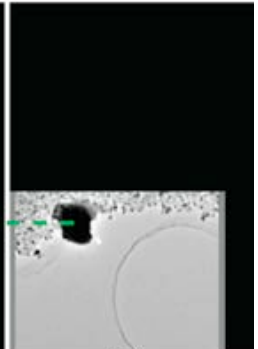

(f)

Figure 2. Sequential frames of video from an in situ heating experiment are shown in (a) - (c). After applying a spatial drift correction and padding the extra space around the frame, the feature of interest remains stationary in adjacent frames, (d) - (f). 